\title{
SYMMETRY PRINCIPLES IN PHYSICS
}

\author{
T.D. LEE
}

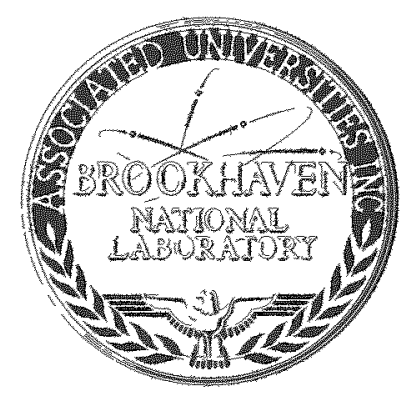

May 1970

\section{BROOKHAVEN NATIONAL LABORATORY} ASSOCIATED UNIVERSITIES, INC. under contract with the UNIED STATES ATOMC ENERGY COMMISSION 


\section{DISCLAIMER}

This report was prepared as an account of work sponsored by an agency of the United States Government. Neither the United States Government nor any agency Thereof, nor any of their employees, makes any warranty, express or implied, or assumes any legal liability or responsibility for the accuracy, completeness, or usefulness of any information, apparatus, product, or process disclosed, or represents that its use would not infringe privately owned rights. Reference herein to any specific commercial product, process, or service by trade name, trademark, manufacturer, or otherwise does not necessarily constitute or imply its endorsement, recommendation, or favoring by the United States Government or any agency thereof. The views and opinions of authors expressed herein do not necessarily state or reflect those of the United States Government or any agency thereof. 


\section{DISCLAIMER}

Portions of this document may be illegible in electronic image products. Images are produced from the best available original document. 
BNL 50261 (T-591)

(Physics - TID-4500)

\title{
SYMMETRY PRINCIPLES IN PHYSICS*
}

\author{
T.D. LEE \\ Columbia University, New York, N.Y.
}

This report was prepared as an account an whork sponsored by the United States Government. Neither the United States nor the United States Atomic Lnergy Commission, nor any of their employes, fior any of their contractors, subcontracturs, or their enployes makes any urranty, express or implied, or assunes any

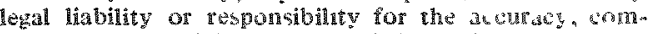

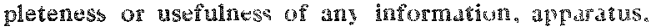

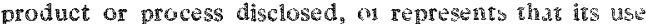
would not inatinge privaty onned righes.

May 1970

*Part of a series of four lectures given at Brookhaven in May 1970.

\section{BROOKHAVEN NATIONAL LABORATORY UPTON, NEW YORK 11973}


NOTICE

This report was prepared as an account of work sponsored by the United States Government. Neither the United States nor the United States Atomic Energy Commission, nor any of their employees. nor any of their contractors, subcontractors, or their employees, makes an warranty, express or implicd, or assumes any legal liability or responsibility for the accurary, completeness or usefulness of any information, apparatus, product or process disclosed, or represents that its use would not infringe privately owned rights.

Printed in the United States of America

Available from

National Technical Information Service

U.S. Department of Commerce

5285 Port Royal Road

Springfield, Virginia 22151

Price: Printed Copy 3.00 : Microfiche $\$ 0.95$

Februar: 1971

1000 copies 


\section{Contents}

1. Introduction

2. Non-observables, Symmetry Transformations and Conservation Laws

3. Asymmetries and Observables

4. Time Reversal

5. Complementarity of Symmetry Violations

6. Aesthetical Considerations 


\section{Introduction}

Symmetry principles have played an important role since the beginning of physics. A great deal of our understanding of nature can be formulated through these symmerry considerations. In this lecture, I would like to review these symmetry operations, and to examine their foundation. Such an examination is useful, especially in view of the various asymmetries that have been discovered during the past two decades.

There are four main groups of symmetries, or broken symmetries, that are found to be of importance in physics.

1. Permutation symmetry: Bose-Einstein and Fermi-Dirac statistics

2. Continuous space-time transformations: translation, rotations, accelerations, erc.

3. Discrete transformations: space inversion $P$, time reversal $T$, particleantipanticle conjugation C, G-parity, etc.

4. Unitary transformations, which include:

$U_{1}$-symmetries: conservation laws of charge $Q$, baryon number $N$, and lepton numbers $L_{e}$ and $L_{\mu}$ " $\mathrm{SU}_{2}$ (isospin) symmetry, and $\mathrm{SU}_{3}$ symmetry.

Among these, the symmetries connected with the first two groups of transformations are, at present, believed to be exact. In the third group only the product CPT is perhaps exact, but each individual discrete symmetry operation is not. In the fourth

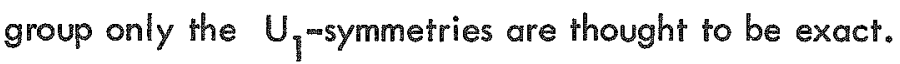


II. Non-Observables, Symmetry Transformations and Conservation Laws

The root of all symmetry principles in physics lies in the assumption that if is impossible to observe certain basic quantities; these will be called "non-observables" in the following. For example, we may consider the interaction energy $V$ between two particles at positions $\vec{r}_{1}$ and $\vec{r}_{2}$. The physical assumption that it is not possible to measure an absolute position leads to the mathematical conclusion that the interaction energy $V$ should be unchanged under a space translation

$$
\vec{r}_{1} \rightarrow \vec{r}_{1}+\vec{\Delta}
$$

and

$$
\vec{r}_{2} \rightarrow \vec{r}_{2}+\vec{\Delta}
$$

Therefore, the interaction energy $V$ is a function only of the relative distance $\left(\vec{r}_{1}-\vec{r}_{2}\right) ;$ i.e.,

$$
V=V\left(\vec{r}_{1}-\vec{r}_{2}\right)
$$

From this, we deduce that the total momentum of this system of two particles must be conserved, since its rate of change is equal to

$$
-\left(\vec{\nabla}_{1}+\vec{\nabla}_{2}\right) V
$$

which, on account of (1), is zero.

This simple example illustrates the close connection berween three aspects of a symmetry principle: the assumption of a non-observable, the implied invariance under the connected mathematical transformation and the physical consequence of a 
conservation law. In an entirely similar way, we assume the absolute time to be a non-observable; the physical laws must then be invariant under a time translation

$$
t \rightarrow t+r
$$

which results in the conservation law of energy. By assuming the absolute spatial direction to be a non-observable, we derive rotation invariance and obtain the conservation law of angular momentum. By assuming that absolute (uniform) velocity is not an observable, one derives the symmetry requirement of Lorentz invariance, and with it the conservation laws connected with the six generators of the Lorentz group. Similarly, the foundation of general relativity rests on the assumption that it is impossible to distinquish the difference between an acceleration and a suitably arranged gravitational field.

The table given on the next page summarizes these three fundamental aspects for some of the symmetry principles used in physics. 


\begin{tabular}{|c|c|c|}
\hline Non-observables & $\begin{array}{l}\text { Symmetry } \\
\text { Transformations }\end{array}$ & $\begin{array}{l}\text { Conservation Laws } \\
\text { or Selection Rules }\end{array}$ \\
\hline $\begin{array}{l}\text { difference between } \\
\text { identical particles }\end{array}$ & permutation & B.E. or F. D. statistics \\
\hline absolute spatial position & space translation $\vec{r} \rightarrow \vec{r}+\vec{\Delta}$ & momentum \\
\hline absolute time & time translation $t \rightarrow+t$ & energy \\
\hline absolute spatial direction & rotation $\hat{r} \rightarrow \hat{r}^{\prime}$ & angular momentum \\
\hline absolute velocity & Lorentz transformation & $\begin{array}{l}\text { generators of the } \\
\text { Lorentz group }\end{array}$ \\
\hline $\begin{array}{l}\text { absolute right } \\
\text { (or absolute left) }\end{array}$ & $\vec{r} \rightarrow-\vec{r}$ & parity \\
\hline $\begin{array}{l}\text { absolute sign } \\
\text { of electric charge }\end{array}$ & $e \rightarrow-e$ (or, $\psi \rightarrow e^{i \phi} \psi^{\dagger}$ ) & $\begin{array}{l}\text { charge conjugation } \\
\text { (or, particle anti= } \\
\text { particle conjugation) }\end{array}$ \\
\hline $\begin{array}{l}\text { relative phase between } \\
\text { states of different } \\
\text { charge } Q\end{array}$ & $\psi \rightarrow e^{i Q \theta} \psi$ & charge \\
\hline $\begin{array}{l}\text { relative phase between } \\
\text { states of different } \\
\text { baryon number } N\end{array}$ & $\psi \rightarrow e^{i N \theta} \psi$ & baryon number \\
\hline $\begin{array}{l}\text { relarive phase between } \\
\text { states of different } \\
\text { lepton number } L\end{array}$ & $\psi \rightarrow e^{i L \theta} \psi$ & lepton number \\
\hline $\begin{array}{l}\text { difference between } \\
\text { different coherent mixture } \\
\text { of } p \text { and } n \text { states }\end{array}$ & $\left(\begin{array}{l}p \\
n\end{array}\right) \rightarrow u\left(\begin{array}{l}p \\
n\end{array}\right)$ & isospin \\
\hline
\end{tabular}




\section{Asymmetries and Observables}

Violations of symmetries arise when what were thought to be non-observables turn out to be actually observables. For example, a proton state and a neutron state are obviously different, since these rwo states carry different electric charges; this observable difference then breaks the isosp in symmetry. As another example, let us consider the questions of right-left symmetry $P$, particle-antiparticle conjugation $C$, and their product $C P$.

Of course, it is well known that even in daily life, right and left are distinct from each other. Our hearts, for example, are usually on our left sides. The word "right" also means correct, while the word "sinister" in its Latin root means left. In

English, one says "right-left", but in Chinese $t / t a ; / I$ (left) always precedes to (right). However, such asymmetry in daily life is attributed to either the accim dental asymmetry of our environment or initial conditions. The same applies also to the difference between particles and antiparticles. Before the discovery of parity nonconservation in 1957, it was assumed that the laws of nature are symmetric under a right=left transformation. The same assumption was made with respect to the particleantiparticle conjugation.

To illustrate these symmetries, or asymmetries, we may imagine two advanced civilizations, completely separate from each other; nevertheless they manage to communicate with each other, but only through neutral unpolarized messages (e.g., unpolarized light). Let us further imagine that through such communications these two civilizations want to reach a mutual agreement on both the convention of the "sign" of an electric charge and the definition of a "right-handed screw". This would not 
be possible, if nature were symmetrical with respect to $C$ and $P$.

However, assuming that these iwo civilizations are as advanced as ours, such an agreement can, in principle, be achieved:

First, both civilizations may perform the three-body decay mode experiments of $K_{L}^{0}$, and compare the decay rates of $e^{+} \pi{ }^{-} v_{\ell}$ and $e^{-} \pi^{+} \bar{v}_{\ell}$. In our convention, we find 1,2

$$
\frac{\operatorname{Rate}\left(K_{L}^{0} \rightarrow \ell^{+} \pi^{-} v_{\ell}\right)}{\operatorname{Rate}\left(K_{L}^{0} \rightarrow \ell^{-} \pi^{+} \bar{v}_{\ell}\right)}= \begin{cases}1.00315 \pm 0.0003 & \text { for } \ell=e \\ 1.00405 \pm 0.00135 & \text { for } \ell=\mu .\end{cases}
$$

Thus, by using a counting device, it is now possible to compare the convention of the sign of electric charge. These slight differences in $K_{\ell 3}^{0}$ decay rates enable one to give an absolute definition of the sign of electric charge, without the use of a test charge.

Once the sign of electric charge is established, it is then possible to transmit the definition of an absolute "right" (or absolute "left") by using $\pi_{\mu 2}^{ \pm}$decay. For example, the neutrino emitted in a $\pi^{+}$decay has its spin always anti-parallel to its momentum, which may be defined to form a "left-handed screw".

We note that by comparing the spin-momentum direction of the antimentrino in $\pi^{-}$decay with that of the neutrino in $\pi^{+}$decay, the $\pi_{\mu 2}^{ \pm}$decay is found to violate $P$ symmetry and $C$ symmetry separately, but not their product $C P$. On the other hand, the $K_{\ell 3}^{0}$ decay violates both $C$ symmetry and $C P$ symmetry, since the total decay rate is unchanged under the space inversion operation. [ The $P$ symmetry is also violated in the $K_{\ell 3}^{0}$ decay, because the neutrino spin is again found to be antim parallel to its momentum. ] 


\section{N. Time Reversal}

If one assumes CPT invariance, then CP asymmetry implies also $T$ asymmetry. Without assuming CPT invariance, we can also deduce by using our present knowledge of $K$-decay that $T$ invariance is violated, provided $^{2}$

$$
\left|\frac{\operatorname{Amp}\left(K_{S}^{0} \rightarrow 3 \pi^{\circ}\right)}{\operatorname{Amp}\left(K_{L}^{0} \rightarrow 3 \pi^{\circ}\right)}\right|<2 .
$$

In this connection, it may be worthwhile to review the meaning of $T$ invarm iance. In classical physics, $T$ invariance implies that if the motion of a system of particles is reversed in time, then the time-reversal sequence is also a possible solum tion of the dynamical equations.

For a macroscopic system with a large number of particles, al though the timereversed sequence is always a possible one if $T$ invariance holds, it is, in general, an improbable one. Thus, if we view a movie showing the motion of any macroscopic system, even if we do nor know for sure whether such a movie is being shown in its timem reversed order or not, we may try to guess. If the number of particles in the system is sufficiently large, our guess will be almost always right. It is in this statistical sense that we can defermine the direction of our macroscopic time.

On the other hand, if the system contains only a very small number of particles, then it is not possible, even in this statistical sense, to differentiate a time-ordered sequence from its time-reversed sequence (provided $T$ invariance holds). As we shall see, this last statement has to be modified in quantum mechanics. 
We may use the $\mu$-decay as an example to illustrate this important difference between the quantum mechanical and the classical rime-reversal invariance for a microscopic system. Consider the decay of, say, $\mu^{-} \rightarrow e^{-}(L)+\vec{v}_{e}(R)+v_{\mu}(L)$ (Fig. 1)

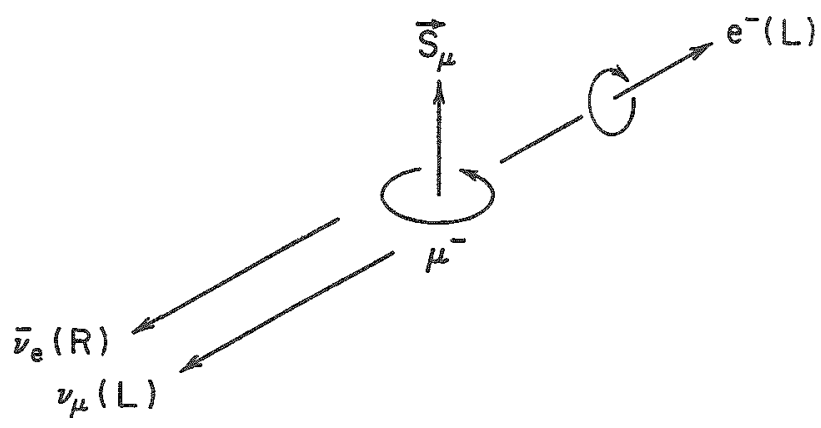

Figure 1

where $\vec{S}_{\mu}$ is the initial spin of $\mu, R$ and $L$ refer, respectively, to a right-hand and left-hand polarization. Now let us examine the time-reversed sequence, and for simplicity we consider only the configurations in which the momenta of $\bar{v}_{e}$ and $v_{\mu}$ are parallel (Fig. 2)

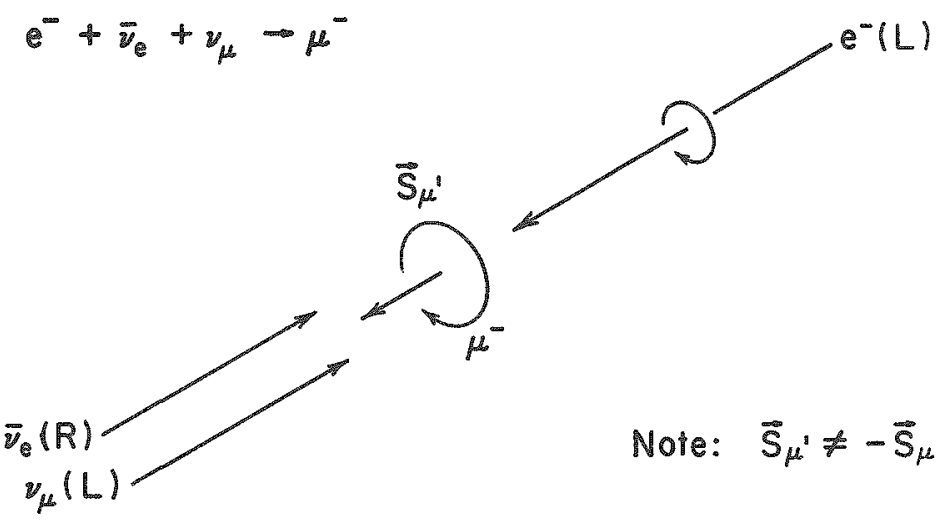

Figure 2 
In $e^{-m}(L)+\vec{v}_{e}(R)+v_{\mu}(L) \rightarrow \mu$, since all initial momenta are assumed to be along the same line, the total angular momentum along this line must be conserved. Thus, the final $\mu$ spin $\vec{S}_{\mu}^{\prime}$ is the same as the initial $\vec{e}$, and $\vec{S}_{\mu}^{\prime} \neq-\vec{S}_{\mu}$. Similar conclusions can be readily derived for the general case when the momenta of the neutrino and the antineutrino are not parallel.

In a corresponding classical physics problem, we should expect the final $\mu$ " spin $\vec{S}_{\mu}^{\prime \prime}$ in the reversed sequence to be the opposite of the initial $\mu \vec{\mu}$ spin $\vec{S}_{\mu}$ in the original sequences. In quantum mechanies, a true time-reversal implies using

$$
T \Psi(t)=U_{T} \psi^{*}(-t)
$$

as the wave function, which implies that for the $\mu$ decay we must simultaneously reverse the $\vec{v}_{e}, v_{\mu}, e \overrightarrow{e m e n t u m ~ a n d ~ s p i n ~ i n ~ a l l ~ p o s s i b l e ~ d i r e c t i o n s ~ a n d ~ c o n s t r u c t ~}$ the required coherent wave; only then, the final $\mu-$ spin would be $-\vec{S}_{\mu}$.

The above considerations can be applied to any reaction

$$
a+b+\ldots \rightarrow a^{d}+b^{\prime}+\ldots .
$$

In quantum mechanics, even for a microscopic system, assuming $T$ invariance once holds, the time reversed solution $T \psi(t)$ is always an improbable one (except for the free particles); it requires the rimemreversed solution to contain, as the initial state, a spherical incoming coherent wave which is extremely difficult to construct. This should not be surprising when one recalls that the degrees of freedom necessary for a quantum mechanical description of a microscopic system are almost always much larger than those of any classical macroscopic system. For all practical purposes, 
a rest of time-reversal invariance is, in quantum mechanics, simply a test of "reciprocity"; time-reversal invariance implies that the reciprocity relation holds.

The matrix element of the S-matrix for reaction (2) is, then, related to that of

$$
a^{i}+b^{d}+\ldots \rightarrow a+b+\ldots
$$

where all particles, $a, b, \ldots$ and $a^{\prime}, b^{\prime}, \ldots$ have definite momenta and helicities: $k_{a} \sigma_{a}, k_{b} \sigma_{b}, \ldots$. We have, if time-reversal invariance holds, the reciprocity relation

$$
\begin{aligned}
& \left|<a^{\prime}\left(k_{a}^{\prime}, \sigma_{a}^{\prime}\right), b^{\prime}\left(k_{b}^{\prime}, \sigma_{b}^{\prime}\right), \ldots\right| s\left|a\left(k_{a}, \sigma_{a}\right), b\left(k_{b}, \sigma_{b}\right), \ldots>\right| \\
& \quad=\left|<a\left(-k_{a},-\sigma_{a}\right), b\left(-k_{b},-\sigma_{b}\right), \ldots\right| s\left|a^{\prime}\left(-k_{a}^{\prime},-\sigma_{a}^{\prime}\right), b^{\prime}\left(-k_{b}^{\prime},-\sigma_{b}^{\prime}\right) \cdots\right\rangle \mid
\end{aligned}
$$

The violation of $T$ invariance means that these reciprocity relations are not valid. 


\section{Complementarity of Symmetry Violations}

Consider the case of parity non-conservation. In the literature, the discussion often proceeds by first defining a space inversion operator $P$ and then producing a Hamiltonian $H$ which does not commute with $P_{*}$ i.e.,

$$
[H, P] \neq 0 \text {. }
$$

It is important to note that this process is not self-consistent. To see this, let us recall that the space inversion operator $P$ is a unitary operator in the Hilbert space and, by definition, it should represent the coordinate transformation-space inversion,

$$
P: \vec{r} \rightarrow-\vec{r}, t \rightarrow++
$$

The time translation operator is

$$
e^{-i H \tau}: \vec{r} \rightarrow+\vec{r}, t \rightarrow++\tau
$$

For the geometrical transformation, it is obvious that the space inversion $\vec{r} \rightarrow-\vec{r}$ must commute with the time translation $\rightarrow+t+\tau$, independently of whether parity is conserved or not. On the other hand, $[H, P] \neq 0$ implies that the alleged space inversion operator $P$ fails to satisfy the multiplication law of the coordinate transformations that it is supposed to represent; thus, the fact that the space inversion symmetry is being violated shows that the unitary operator $P$ is not defined.

It is, however, possible to give a definition of $\mathrm{P}$ by making an approximation on $H$. We may replace the total Hamiltonian by, say, $H_{i}$ (e.g., $H_{i}$ may denote either the strong, or the electromagnetic, or the weak interaction Hamiltonian), such 
that within this approximation, a parity operator $P=P_{i}$ can be defined and it commutes with $H_{i}$,

$$
\left[H_{i}, P_{i}\right]=0 \text {. }
$$

It is clear that such an approximate definition $P_{i}$ depends on the particular interaction $H_{i}$ that is being chosen. 3

To illustrate this interaction-dependent nature of $P_{i}$, let us consider the well known 0 -r puzzle:

$$
K^{+} \rightarrow \begin{cases}\pi^{+} \pi^{0} & (\theta \text {-mode }) \\ \pi^{+} \pi^{0} \pi^{0} & (\tau \text {-mode })\end{cases}
$$

It is known from the strong $\pi \mathrm{N}$ interaction that the pion is a pseudo-scalar; the same conclusion is also reached by studying the electromagnetic decay of $\pi^{\circ}$,

$$
\pi^{\circ} \rightarrow 2 \gamma \rightarrow 2 e^{+}+2 e^{-}
$$

Thus, we have

$$
P_{s t}\left(\pi^{0}\right)=P_{\gamma}\left(\pi^{0}\right)=-1
$$

and consequently the parity difference berween $\theta$ and $T$ :

$$
P_{i}(\tau)=P_{i}(\theta) \cdot P_{i}(\pi)=-P_{i}(\theta)
$$

where the subscript i denotes either "st" or " $\gamma$ ", indicaring that the parity is determined by either the strong or the electromagnetic interaction.

On the other hand, we can just as well try to use the weak and $\tau$ decays to determine the parity, $\mathrm{P}_{\text {wk }}$. One has then, by definition, 


$$
P_{w k}(\theta)=P_{w k}(T)
$$

and, therefore,

$$
P_{w k}\left(\pi^{0}\right)=+1
$$

Clearly, $\left[\mathrm{H}_{s t}, \mathrm{P}_{w k}\right] \neq 0$ and $\left[\mathrm{H}_{\gamma}, \mathrm{P}_{w k}\right] \neq 0$. The violation of parity can then be viewed as either the weak interaction violating the parity determined by the strong (and the electromagnetic) inferaction, or alternatively, as the strong (and the electromagnetic) interaction violating the parity determined by the weak interaction.

In practice, since $\mathrm{H}_{s f}$ and $\mathrm{H}_{\gamma}$ are much stronger than $\mathrm{H}_{w k}$, it is more convenient to define $P$ by $H_{s t}$ and $H_{\gamma}$, and to regard its violation as due to $H_{\text {wk }}$. This then leads to the conventional concept of small symmetry violations. Identical considerations can be extended to other symmetry violations such as $C, C P$ and $T$. 


\section{Aesthetical Considerations}

These recent discoveries of asymmetries raise many interesting problems; some of these are not rotally connected with physics, but rather with our aesthetical considerations. Why do natural laws have a connection with symmetries, yet often with a slight amount of asymmetry rather than with perfect symmetry? This situation may appear to some people as aesthefically disturbing. Why should nature be slightly asymmetrical?

Before discussing these illusive philosophical implications, let us recall that the word "symmetry" has two different meanings even in our daily language. According to the (1949) Webster's Dictionary, these two definitions are:

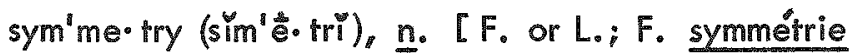
(now symétrie), fr. L., $\overline{f r}_{0}$ Gr. symmetria, fr. synt metron, a measure. I 1. Now Rare. Due or balanced proportions; beauty of form arising from such harmony. 2. Correspondence in size, shape, and relative position, of parts that are on opposite sides of a dividing line or median plane.

It is of interest to note the italic Now Rare for the first definition. Can it be anticipating the broken symmetry principles that physicists have now discovered about nature? Indeed, as we shall see, perhaps beauty should be associated with a slight asymmetry, rather than with total symmetry.

The concept of beauty is, of course, quite subjective. Which is a more beautiful object, one with total symmetry, or one with a slight asymmetry? The answer is clearly open to debate. However, we may take a look at some of the well known examm ples in art. For example, both the Greek statues in Figs. 3 and 4 emphasize bilateral 
symmetry. The painting of Poplars by Monet in Fig. 5 suggests a discrete spacem translational symmetry. While the beauty of symmetry is clearly demonstrated in each case, this beauty is greatly enhanced by the presence of slight asymmetries.

As we have discussed, the validity of all symmetry principles rests on the theoretical hypotheses of non-observables. Some of these hypotheses may indeed be correct in a fundamental sense, some may simply be due to the limitations in our present abilities to measure things. As we improve our experimental techniques, our domain of observations naturally becomes enlarged. It should not be surprising that we may even succeed in observing some of those supposed non-observables, and therein lies the root of symmetry breaking.

In this sense, we should be prepared for the eventual possibility that we might be able, in some distant future, to measure the absolute space-time position, the absolute direction and the absolute velocity, the relative phase between two states of different lepton numbers, or even of different baryon numbers, or of different charges. Even if these were possible, it should be expected that such discoveries could lead only to what appears to be small symmetry breakings, at least in all presently known phenomena, because, otherwise, these supposed non-observables would have been observed long ago: Just as in most of our artistic creations, the harmony and beauty of symmetry is always enhanced by the presence of a small degree of asymmetry. From an aestheric point of view, I think it is rather satisfying to find nature also has a similar preference in small symmetry violations. 


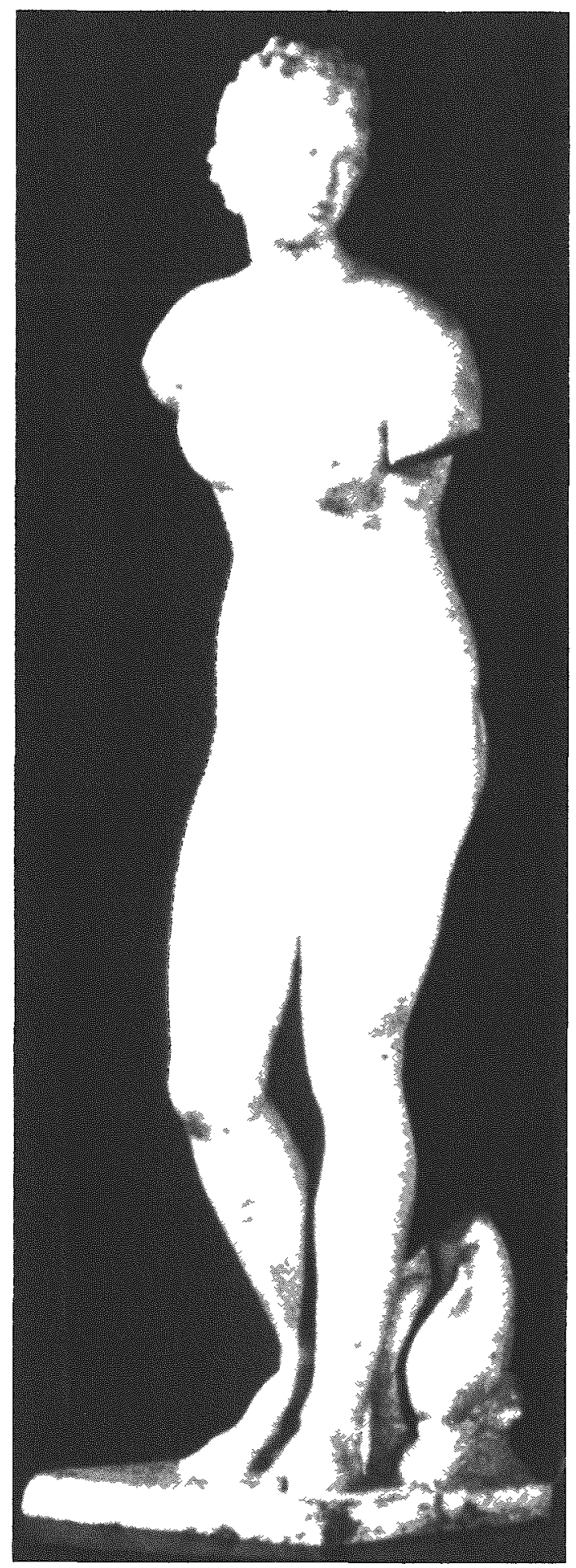

Fig. 3

$-16=$ 


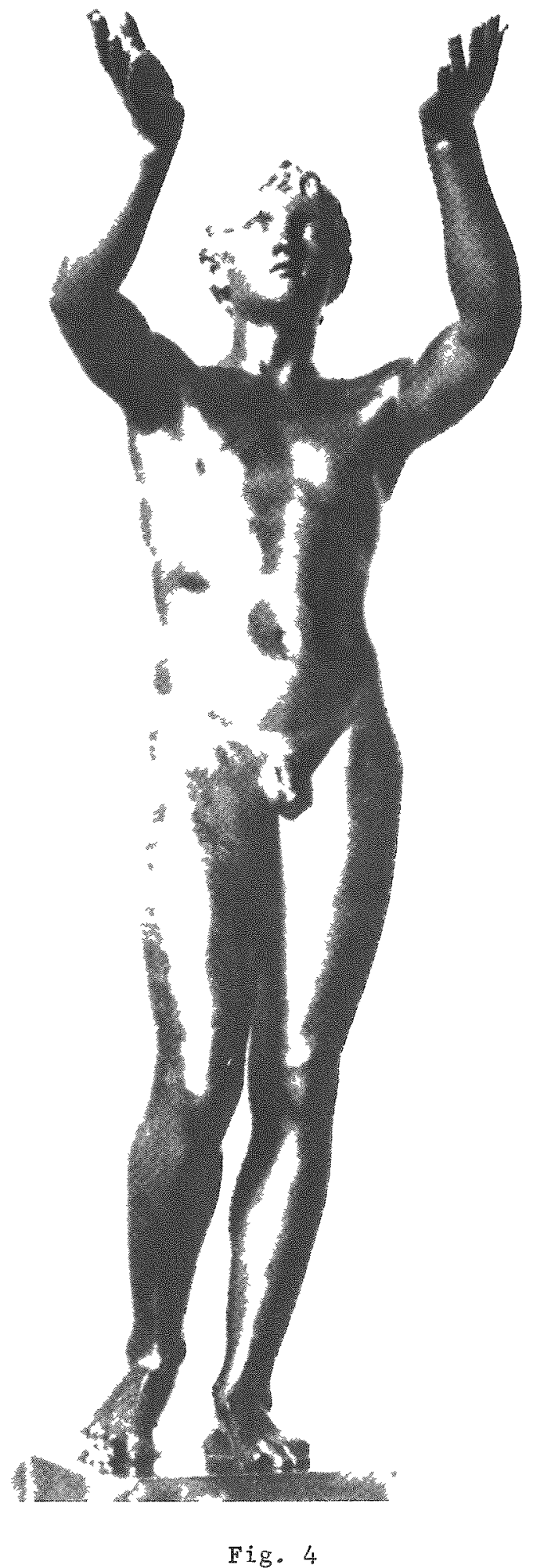

$-17$. 


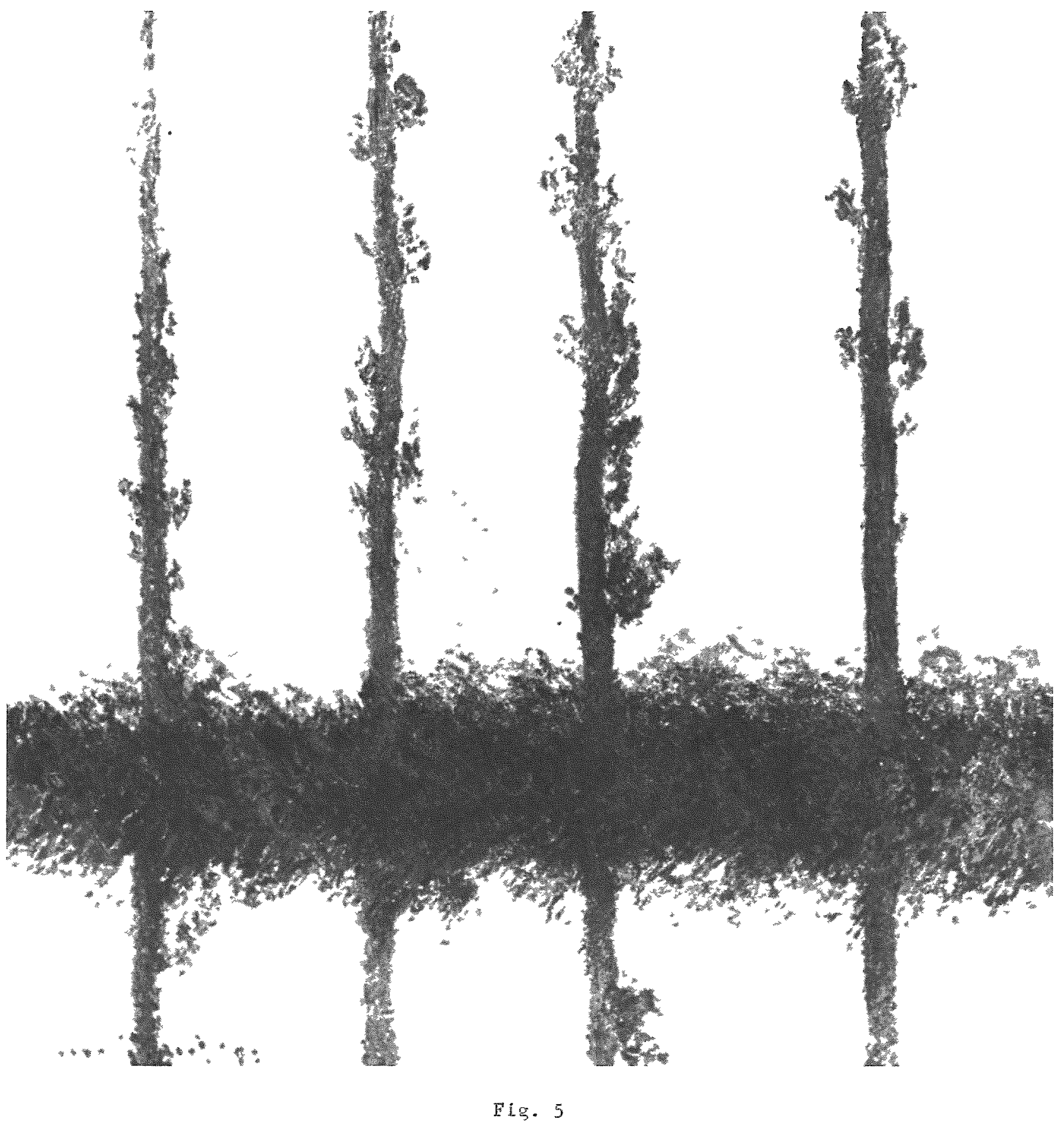




\section{References}

1. D. Dorfan et al., Phys. Rev. Letters 19, 987 (1967).

S. Bennett et al., Phys. Rev. Letters 19, 993 (1967).

2. J. Steinberger, Topical Conference on Weak Interactions (CERN, 1969), p. 291.

3. For a general discussion, see T. D. Lee and G. C. Wick, Phys. Rev. 148, 1385 (1966). 